\title{
Plain X-ray can be a Good Tool for the Diagnosis of Horse Shoe Kidney
}

\author{
MM RAHMAN
}

(J Bangladesh Coll Phys Surg 2012; 30: 56-57)

A 32 year-old male presented with non specific abdominal pain for last five years, diffuse swelling lower abdomen, and recurrent attacks of burning micturitation and passage of stone per urethra once. Plain Xray KUB showed multiple round to oval radio opaque shadows suggestive of urinary stones along the upper pelvis and lower abdomen. Ultrasonography and related investigations(IVU) settled it as Horse shoe kidney. The plain X-ray beautifully demonstrated as the stones maintained the line of a horse shoe kidney.

Most of the time, a horseshoe kidney is an incidental finding on an examination for some other condition that the patient is having. But once it is discovered, there are many options for imaging the anomaly. The kidneys

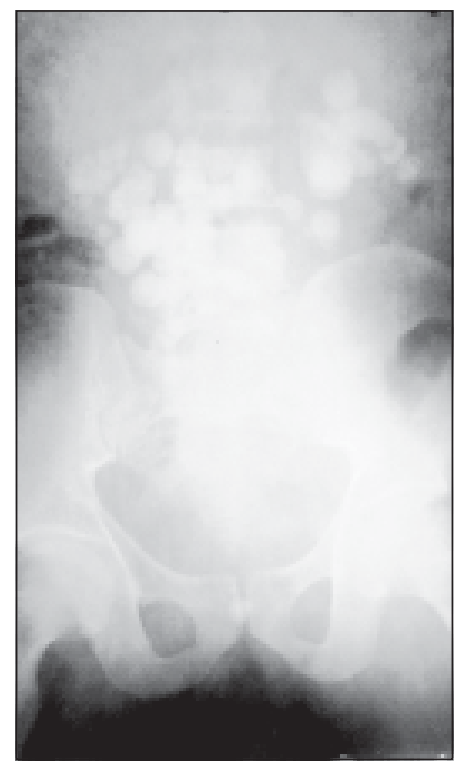

A. Plain X ray KUB shows palisades of radioopaque shadows of different sizes suggestive of stones maintaining the shape of horse shoe kidney.

Prof. Md. Mizanur Rahman, Professor, Dept. of Surgical Oncology, National Institute of Cancer Research and Hospital, Mohakhali, Dhaka. can be seen on plain radiographs, but the definition is not as clear as in some of the other modalities. They will be discovered on plain radiographs because of their lower location and the location of the lower poles being more medially rotated than would be expected, ureters as well, to see if there are any abnormalities lower in the system.

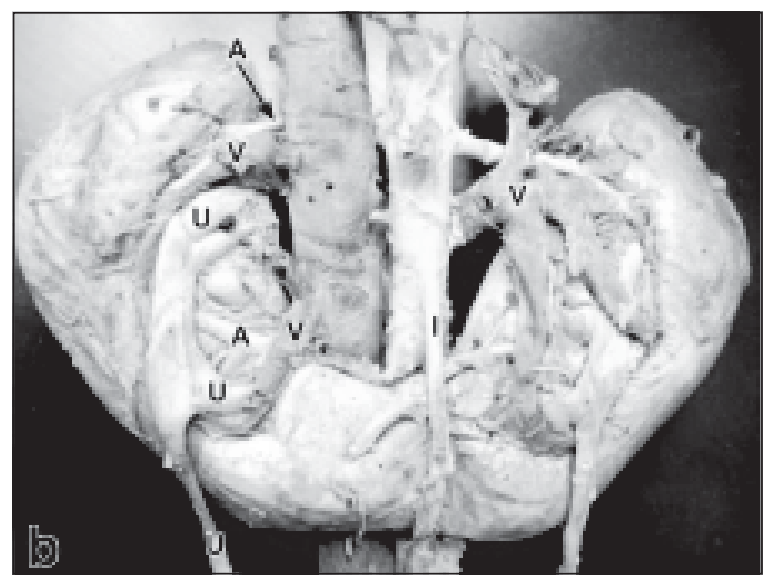

B. Horseshoe kidney shown with descending aorta and inferior vena cava.

Note. From "A horseshoe kidney with partial duplex systems,” by K. Ongeti, J. Ongeng, and H. Saidi 2011, International Journal of Anatomical Variations, 4, p 56.

Horseshoe kidney is the most common fusion anomaly in the kidney and occurs in about 1 in 400 people, or about $25 \%$. It is also twice as likely to occur in males as in females ${ }^{1}$. Although it is not highly common, it isn't uncommon either. Normally one-third of the patients that have horseshoe kidney are asymptomatic and the condition is noticed incidentally on radiologic examination 2. Although most patients are 
asymptomatic, there are certain conditions that go with horseshoe kidney quite frequently.

\section{Anatomy of the Horseshoe Kidney}

Horseshoed kidney is the most common type of fusion anomaly. For the most part, the horseshoe kidney functions as a normal kidney. Many times, kidney malformations are accompanied by lower urinary tract anomalies as well. This is understandable because the kidney and the ureter arise from the same single embryonic structure ${ }^{3}$. With horseshoe kidney, the kidneys can be located anywhere along the normal embryologic ascent of the kidneys. Normally they are located lower in the pelvic region of the body. In 90\% of cases, the fusion of the kidneys occurs in the lower poles. In this condition, both kidneys are malrotated and their lower poles are joined. In this condition, both kidneys are malrotated and their lower poles are joined. With the fusion, there is an isthmus that crosses the midline of the body to connect the two kidneys.

\section{Symptoms and Complications:}

It is also twice as likely to occur in males than in females ${ }^{4}$, Normally one-third of the patients that have horseshoe kidney are asymptomatic, and the condition is noticed incidentally on radiologic examination ${ }^{2}$. Although most patients are asymptomatic, there are certain conditions that go with horseshoe kidney quite frequently.

When symptoms are present, they are usually because of obstruction, stones or infection with urinary tract infection being the most common presenting symptom in children" ${ }^{1}$. Then next most common occurrence is presence of kidney stones. Kidney stones will develop in anywhere from $20 \%-60 \%$ of patients. Kidney stones go hand-in-hand with obstructions; they have a tendency to cause one another.

\section{References:}

1. O’Brien, J., Buckley, O., Doody, O., Ward, E., Persaud, T., \& Torreggiani, W. (2008). Imaging of horseshoe kidney and their complications. Journal Of Medical Imaging And Radiation Oncology, 52(3), 216-226.

2. Khan, A., Myatt, A., Palit, V., \& Biyani, C. (2011). Laparoscopic heminephrectomy of a horseshoe kidney. Journal of the Society of Laparoendoscopic Surgeons, 15(3), 415-420.

3. Adalat, S., Bockenhauer, D., Ledermann, S., Hennekam, R., \& Woolf, A. (2010). Renal malformations associated with mutations of developmental genes: messages from the clinic. Pediatric Nephrology, 25(11), 2247-2255.

4. Ongeti, K. W., Ogeng’o, J. \& Saidi, H. (2011). A horseshoe kidney with partial duplex systems. International Journal of Anatomical Variations, 4, 55-56. 